\title{
Shifts of attention in the identification and discrimination of intensity
}

\author{
ROBERT M. NOSOFSKY \\ Harvard University, Cambridge, Massachusetts
}

\begin{abstract}
Subjects' ability to resolve stimuli varying in loudness is studied by manipulating task requirements and sequential dependencies in signal presentations. In the first experiment, subjects are required both to identify and to discriminate the same set of 11 randomly presented signals. On the one hand, resolution performance is virtually unchanged under the differing task instructions. On the other hand, large effects on sensitivity are obtained in Experiments 1, 2 , and 3 in both identification and discrimination tasks when sequential dependencies are manipulated. The results are interpreted in terms of a shifting attention band in the intensity dimension, as suggested by Luce, Green, and Weber (1976). Preliminary evidence that subjects can voluntarily focus the attention band on a given region of the intensity continuum is obtained.
\end{abstract}

Two signals separated by $5 \mathrm{~dB}$ are almost perfectly identifiable in a two-alternative loudness absolute-identification (AI) task. Yet the same two signals are badly confused when embedded in a multisignal AI task. A common explanation is that the phenomenon is the result of poor perceptual memory for unidimensional "sensory" stimuli. In particular, $n$ memory representations must be maintained simultaneously in an n-alternative AI task, and the subject may be capacity-limited as $n$ becomes larger. Furthermore, in the two-alternative AI task, each signal presentation serves as a memory standard upon which the next signal presentation may be judged. In an n-alternative AI task, the retention interval between successive presentations of a given signal will generally be longer than in the two-alternative situation (Siegel, 1972).

Explanations based solely on the memory-retention interval run into trouble, however, when absolute identification performance is examined in more detail. Purks, Callahan, Braida, and Durlach (1980) found that subjects' sensitivity in distinguishing between a given pair of signals (measured in $d^{\prime}$ ) was independent of the signal presented on the preceding trial. In particular, subjects were no better than average at distinguishing between signals $i$ and $i+1$ when one of these signals was presented on the preceding trial. Luce, Nosofsky, Green, and Smith (1982) found only slight improvements in sensitivity in a similar analysis.

This work was supported in part by National Science Foundation grants to Harvard University. I would like to thank David $M$. Green and R. Duncan Luce for useful discussions and suggestions and for their comments on an earlier version of this article. My mailing address is: Department of Psychology, Harvard University, Cambridge, Massachusetts 02138.
A second phenomenon not easily explained by the retention interval hypothesis is the relationship observed between identification performance and the range of the stimulus set. If one holds constant the number of equally spaced stimuli and increases the range that they occupy, performance does not improve nearly as much as the Thurstonian model predicts (Braida \& Durlach, 1972; Pollack, 1952). The range phenomenon can be viewed more directly in another experimental manipulation. Two stimuli whose locations remain fixed are less identifiable when the remaining stimuli in the AI task encompass a wide range than when the range is narrow (Gravetter \& Lockhead, 1973; Hartman, 1954). Why increasing stimulus range should result in memory deterioration is not obvious. In particular, the number of memory representations that a subject must maintain remains fixed, and the average length of the retention interval is unchanged.

To account for the relationships in resolution performance observed between different psychophysical tasks, Durlach and Braida (1969) proposed that the subject operates in one of two distinct memory modes. The use of these modes depends on the task requirements of a given experiment. In the sensorytrace mode, the subject tries actively to maintain the traces of past stimulus presentations. This mode of memory operation is assumed to be used in discrimination experiments. Memory noise in the sensorytrace mode increases with time delay and interference, but is otherwise independent of the experimental context. In the context-coding mode, the subject attempts to determine the relationship of a given sensation to the general context of other sensations that occur in the experiment. Noise effects in the context-coding mode are assumed to be independent of time, but to depend directly on the range of the stimulus set, with larger ranges leading to more 
noise. Durlach and Braida assumed that only the context-coding mode of memory operation is used in standard AI tasks. The theory is therefore able to explain (among other things) the relationship between stimulus range and identification performance, as well as why identification performance depends little on the preceding signal (Purks et al., 1980). A similar theory relating range to identification performance was proposed independently by Gravetter and Lockhead (1973), although this theory stressed that criterial range (i.e., the range between the two extreme category boundaries) was the relevant factor.

Whereas the range theories have stressed memory and judgmental limitations in absolute identification, an alternative approach that stresses attentional limitations has been suggested by Luce, Green, and Weber (1976). Thus far, the theory has been applied mainly in the domain of loudness identification. In brief, the attention-band theory (Luce et al., 1976) posits a mechanism of selective intensity attention that monitors a relatively narrow intensity range. Signal presentations that fall outside the attention band are only partially monitored by the central nervous system, and so their internal representations will be much more variable. As one increases stimulus range, the probability of a signal's falling within the band is diminished, and sensation noise will generally increase.

As a secondary hypothesis, it was assumed that the attention band would track signal presentations probabilistically. Indeed, it would make little sense for a selective attention mechanism to exist if it did not monitor relevant information channels. The finding by Purks et al. (1980), however, that sensitivity is independent of the last signal, causes the attention-band hypothesis trouble. Nevertheless, it is possible that in order to observe sequential effects in sensitivity, the sequential dependencies in signal presentations need to be made more extreme.

To test the attention-band hypothesis and simultaneously rule out theories positing that the variability of the signal representations in $\mathrm{AI}$ is a function solely of range, the following experiment was devised by Luce et al. (1982). In one condition, termed the random-step condition, a standard nalternative AI experiment was run. In particular, there were 11 signals equally spaced in decibels, spanning a 40-90-dB range, and the signal selected for presentation on a given trial was random. In a second condition, termed the small-step condition, the same signal set was used, but the sequential dependencies. in signal presentations were constrained. In particular, on each trial the signal selected for presentation was equally likely to be the same one just presented, the one just above it, or the one just below it. It should be noted that, asymptotically, every signal is presented equally often in the small-step condition. Thus, the random- step and small-step conditions are equated with respect to overall range and asymptotic signal presentation probability-only the sequential dependencies in signal presentations are manipulated.

Luce et al. (1982) found vastly improved sensitivity in subjects' identification performance in the small-step condition relative to the random-step condition. ${ }^{1}$ The finding lent support to the attentionband theory, and also seemed to rule out hypotheses suggesting that the variance of signal representations in AI tasks is a function solely of stimulus range. Nevertheless, there are strong reasons to question the meaningfulness of the result. The small-step condition seems to be different in principle from a standard AI task. In particular, it seems likely that the subject may have been employing a discrimination strategy in performing this task, rather than actually "identifying" the stimuli. For example, if signal 5 was presented on trial $n$, and the subject judged the signal on trial $n+1$ to be louder, he or she would respond 6; if the signal on trial $n+1$ was judged to be the same as the signal on trial $n$, the subject would respond 5 , etc. Since subjects were required only to make these types of directional judgments, they may have been operating in the sensory-trace mode of memory operation posited by Durlach and Braida.

This hypothesis receives some support when it is recalled that performance on a standard randomstep AI experiment depends little on the preceding signal. Although Luce et al. (1982) found slightly improved sensitivity in subjects' ability to distinguish signals $i$ and $i+1$ when one of these signals was presented on the preceding trial in the random-step condition, performance was not nearly as good as in the small-step condition-this, despite the same one-trial sequential dependencies. This result gives further reason to believe that different higher level strategies or processes were being used in the two different conditions.

In summary, there are at least two explanations for subjects' sensitivity in the small-step condition being vastly superior to their sensitivity in the random-step condition. One hypothesis is that some cumulative aspect of the sequential dependencies in signal presentations improved sensitivity-for example, by allowing attention to remain fixed in a given intensity region, or to shift gradually along the intensity continuum. A second hypothesis is that improved sensitivity in the small-step condition was the result of changed task requirements-in particular, subjects were able to use a discrimination strategy in the small-step experiment that was not used in the random-step design.

\section{EXPERIMENT 1}

The main purpose of Experiment 1 was to distinguish between these competing hypotheses ex- 
plicitly. In order to do so, the random-step and small-step conditions were replicated. In addition, the following condition was introduced. The same signal set was employed, and signal presentations were random, but the subjects were now required to discriminate successive presentations of stimuli rather than to identify them. That is, the subjects were required to judge only whether the signal on trial $n+1$ was softer than, the same as, or louder than the signal on trial $\mathrm{n}$. This condition was referred to as the discrimination condition. Thus, the random-step and discrimination conditions were identical with respect to signal presentations-only the task requirements were changed. In the discrimination condition, the subject needed only to maintain a single memory representation at any one time. The subject could actively rehearse and maintain the trace of the last signal presented, and use it as a standard upon which to base his or her next response.

It should be noted that most of the trials in the discrimination condition were trivially easy. For example, a soft signal might be presented on trial $n$, and then a loud signal on trial $n+1$. The particular trials of interest were those that corresponded to the ones that occur in the small-step designnamely, those trials in which the same signal was presented successively, or in which two adjacent signals were presented successively. The subjects' ability to resolve signals $i$ and $i+1$ was compared across the three experimental conditions, contingent on the preceding signal's being either $i$ or $i+1$. If performance in the discrimination condition was virtually the same as in the small-step condition, it would be most reasonable to conclude that improved sensitivity in the small-step schedule relative to the randomstep was the result of the changed task requirements. If performance in the discrimination condition was virtually the same as in the random-step condition, it would be most reasonable to conclude that the cumulative nature of the sequential dependencies in the small-step condition was responsible for heightened sensitivity. If performance in the discrimination condition was intermediate between the small-step and random-step conditions, it would be most reasonable to conclude that both factors were important.

\section{Method}

The signals, wide-band noise gated on for $500 \mathrm{msec}$, were presented binaurally, in quiet, via TDH-50 headphones. The observers, one male and one female, were tested in single-wall sound-treated chambers (IAC-402A). Blocks of 100 trials were run, with rests between blocks. Approximately 10 blocks of trials were run per daily 2 -h session.

The stimulus set always consisted of 11 signals spaced at intervals of $5 \mathrm{~dB}$ from 40 to $90 \mathrm{~dB}$ SPL. In the random-step and discrimination conditions, successive signals were equally likely and were selected at random, independently of previous choices. In the small-step condition, a Markov transition matrix was used to program the selection of successive signals. On the first trial of each block, the signal selected for presentation was random. Assume that signal $i$ was presented on trial $n_{;}$then on trial $n+1$, there was a $1 / 3$ probability of signal $i-1$ 's being presented, a $1 / 3$ probability of signal i's being presented, and a $1 / 3$ probability of signal $i+1$ 's being presented. For the edge signals ( 1 and 11 ), there was a $2 / 3$ probability of the signal's being repeated and a $1 / 3$ probability of the adjacent signal's being presented. This Markov chain has the property that, asymptotically, every stimulus is used equally often. ${ }^{2}$

The order of conditions tested was random-step, small-step, and discrimination. For each subject, approximately 5,500 trials were collected in the random-step condition, 4,000 trials in the small-step condition, and 6,700 trials in the discrimination condition. The first 500 trials of each condition were considered practice and were not included in the analyses

At the beginning of each block of trials, the ascending sequence of intensities was played twice. During these presentations, the identity of each signal was printed on the terminal. In the identification conditions (random-step and small-step), the observers were instructed to use the numbers from 1 (softest) through 11 (loudest) as responses to the signals. In the discrimination condition, the subjects were instructed to enter a 1 if the signal on trial $n+1$ was softer than the signal on trial $n$, to enter a 2 if the signal on trial $n+1$ was louder than the signal on trial $n$, and to enter a 3 if the signal on trial $n+1$ was the same as the signal on trial $n$. The observers were encouraged to respond accurately in all conditions. At the end of each block in the identification conditions, the subjects were presented with a summary of their percent correct on each signal and their overall percent correct for the block. At the end of each block in the discrimination condition, the subjects were presented with two summary measures-their overall percent correct for the entire block, and their overall percent correct on those trials in which successive presentations were separated by less than or equal to one step.

At the beginning of each trial, the word "READY" appeared on the terminal for $500 \mathrm{msec}$. After the signal had been presented, the observer entered a response; the number appeared on the screen and could be corrected prior to its being entered into the computer (PDP-15). After both observers had responded on a trial, the correct answer was provided and the next signal was presented following a 500 -msec delay.

The observers were paid \$3.00 per hour plus a \$0.75 per hour bonus for completing the entire experiment.

\section{Results and Discussion}

Throughout this section, the subjects' ability to resolve signals $i$ and $i+1$ is measured in 'd'. The reader should note that the definitions that follow are always in reference to a fixed signal pair, $(i, i+1)$. In the identification conditions, $d^{\prime}{ }_{i, i+1}$ is calculated as follows. Whenever stimulus $i+1$ is presented and a subject responds $i+1$ or greater, it is considered a "hit," whereas a response of $i$ or less is considered a "miss." Similarly, whenever stimulus $i$ is presented and a subject responds $i+1$ or greater, it is considered a "false alarm," whereas a response of $i$ or less is a "correct rejection."

An analogous procedure is used to calculate $d^{\prime}{ }_{i, i+1}$ in the discrimination condition. There are two main cases to consider in the calculation of each $d^{\prime}{ }_{i, i+1}$ pair. First, assume that stimulus $i$ is presented on trial $n$. If stimulus $i+1$ is presented on trial $n+1$ 
and the subject responds "louder," it is considered a "hit," whereas a response of "same" or "softer" is considered a "miss"; if stimulus $i$ is presented on trial $\mathrm{n}+1$ and the subject responds "louder," it is considered a "false alarm," whereas a response of "same" or "softer" is a "correct rejection." Second, assume that stimulus $i+1$ is presented on trial $n$. If stimulus $i+1$ is presented on trial $n+1$ and the subject responds "same" or "louder," it is considered a "hit," whereas a response of "softer" is a "miss"; if stimulus $\mathrm{i}$ is presented on trial $\mathrm{n}+1$ and the subject responds "same" or "louder," it is considered a "false alarm," whereas a response of "softer"' is a "correct rejection." 3

The values of $d^{\prime}{ }_{i, i+1}$ obtained in each condition are plotted in Figure 1. These are based on the data obtained after cumulating over the S-R matrices of the individual subjects. Both subjects showed a similar pattern of results. Consider first the solidline curves. These are values of $d^{\prime}{ }_{i, i+1}$ obtained in the identification conditions. The filled triangles are values of $d^{\prime}{ }_{i, i+1}$ in the random-step condition when the previous signal was either $i$ or $i+1$. (Separate $d^{\prime}$ values were computed for each prior signal and then averaged together.) Similarly, the filled diamonds are the values of $d^{\prime}{ }_{i, i+1}$ in the small-step condition when the previous signal was (necessarily) $i$ or $i+1$. The subjects never made an error on stim-

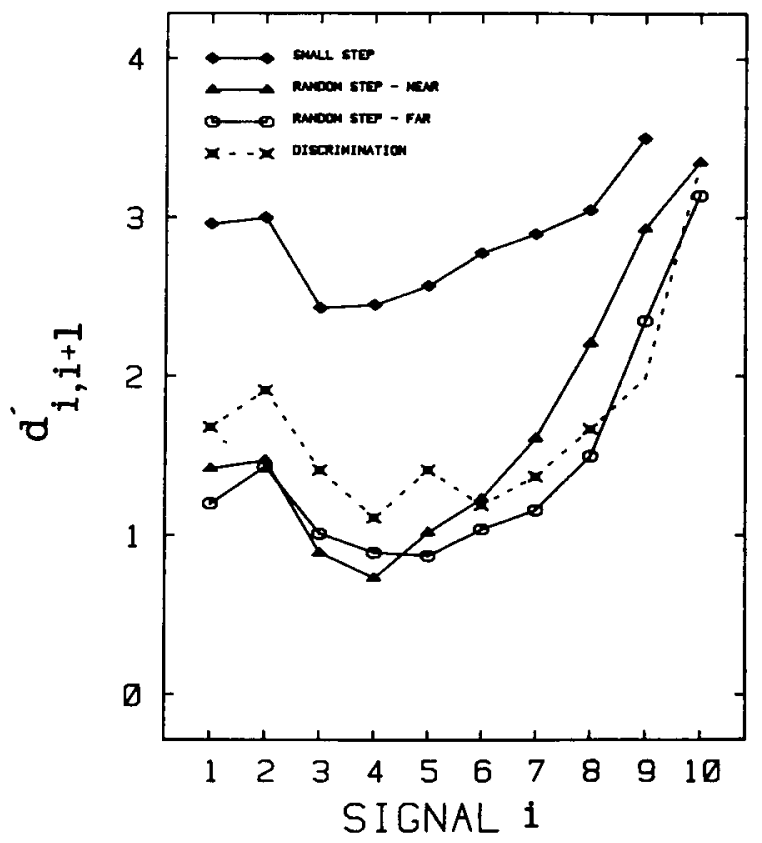

Figure 1. Valiew of $d^{\prime}{ }_{1,1+1}$ as a function of aignal if for the smallstep condition, the random-step condition when the prevlous signal was near, the random-atep condition when the previous signal was far, and the discrimination condition. Only a single d' estimate was avaliable for $d$ ', 10 and $d^{\prime} 10,11$ in the discrimination condition, due to empty cells in the S-R matrix. This is indicated in the figure by the miasing symbols. ulus 11 in this condition, and so an estimate of $d^{\prime} 10,11$ was unavailable. The open circles are the $\mathrm{d}^{\prime}{ }_{\mathrm{i}, \mathrm{i}+1}$ values in the random-step condition when the previous signal was at least two signals away from the closer signal in the pair. For example, the overall $d^{\prime}{ }_{4,5}$ is computed by averaging over the $d^{\prime}{ }_{4,5}$ values obtained when the previous signal was either 1,2 , $7,8,9,10$, or 11 .

These results replicate those already reported in Luce et al. (1982). There is some slight improvement in the subjects' ability to resolve signals $i$ and $i+1$ in the random-step condition when the previous signal is near rather than far from the current signal. This result is significant by a Wilcoxon test $[\mathrm{W}(10)=6, \mathrm{p}<.05$, nondirectional]. There is vast improvement in the subjects' ability to resolve signals $i$ and $i+1$ in the small-step condition.

The values of $d^{\prime}{ }_{i, i+1}$ obtained in the discrimination condition are plotted along the dashed-line curve. These results are similar to those obtained in the random-step condition when the previous signal was either $i$ or $i+1$, and are not at all similar to those obtained in the small-step condition. One may rule out the hypothesis that the subjects' improved sensitivity in the small-step condition was the result solely of a discrimination strategy. It seems reasonable to conclude that the cumulative nature of the sequential dependencies in the small-step condition was of fundamental importance in improving the subjects' sensitivity in that condition. The result obtained in the discrimination condition makes the small-step condition result all the more impressive.

The comparison of performance in the discrimination condition with performance in the random-step condition is interesting in its own right, aside from the inferences it allows one to draw regarding the subjects' small-step performance. It is difficult to conclude definitively whether or not there is any improvement in resolution performance in the discrimination condition relative to the random-step condition (when the previous signal was either $i$ or $i+1)$. Sensitivity seems slightly improved on the soft side of the intensity continuum, but reduced on the loud side. Both subjects showed this pattern of results. It should be noted that any practice effects that may have influenced performance would have favored the discrimination condition performance, since this was the last condition tested. It seems safe to conclude that whatever gains in resolving power there may have been in the discrimination condition relative to the random-step condition were slight. This result was surprising to me, since I had expected that the reduced memory load of the discrimination condition would greatly improve the subjects' ability to resolve adjacent signals.

Two-trial sequential dependencies. The factor of fundamental importance in improving the subjects' sensitivity in the small-step condition appears to be the 
cumulative nature of the sequential dependencies in signal presentations. The subjects' sensitivity in resolving signals $i$ and $i+1$ in the random-step condition and the discrimination condition when the previous signal is $\mathbf{i}$ or $\mathbf{i}+1$ is not nearly as good as in the small-step condition despite the same onetrial sequential dependencies. If the cumulative nature of the sequential dependencies is the crucial factor, one might expect to see additional differences in resolving power in the random-step and discrimination conditions if sequential dependencies further back than one trial are examined.

To explore this issue in some depth, additional data that were obtained in a second experiment with the same subjects are introduced in this section. (These new data are also reported in Experiment 2 of this article, for different purposes.) The new data are from a second random-step AI experiment using the same signal set as reported previously. Each subject made 3,800 responses, all of which are included in the analyses. It should be noted that both subjects' performance improved in the second randomstep AI experiment (RS2) relative to the first (RS1), and that a similar pattern of one-trial sequential dependencies was observed.

Let $S_{n}$ denote the signal presented on trial $n$. All trials selected for the present analysis satisfy the constraint that $\left|S_{n}-S_{n-1}\right|<1$. The data are partitioned, however, according to the signal presented on trial $\mathbf{n - 2}$. One set of data satisfies the constraint that $\left|S_{n-1}-S_{n-2}\right|<1$. For the other set of data, we have $\left|S_{n-1}-S_{n-2}\right|>2$.
The results of the analyses are presented in Figure 2. The reader should note that a different method of analysis was used in RS2 than was used in the other two conditions. Although there is no observable effect of $S_{n-2}$ on sensitivity in RS1, a clear effect emerges in the discrimination condition and in RS2. The results for the latter two conditions provide converging evidence that it is the cumulative nature of the sequential dependencies in the smallstep condition that is responsible for heightened sensitivity.

Summary. In summary, the subjects' sensitivity in resolving adjacent stimuli in each of these experiments is influenced by sequential dependencies in the signals presented. It seems to make relatively little difference, however, whether subjects are required to "identify" signals or to "discriminate" successive signals. The results are consistent with the attentionband theory proposed by Luce et al. (1976), and challenge theories that posit that the variance of signal representations in absolute identification is a function solely of stimulus range.

\section{EXPERIMENT 2}

Chase, Bugnacki, Braida, and Durlach (1983) conducted an absolute-identification study in which the a priori probability of signal presentations was manipulated. The signal set consisted of 13 intensities spaced equally in decibels from 42 to $90 \mathrm{~dB}$. In one condition, all intensities were equally likely. In a second condition, the middle intensity was presented

$$
\begin{aligned}
& \left|s_{n}-s_{n-1}\right| \leq 1, \quad\left|s_{n-1}-s_{n-2}\right| \leq 1 \\
& \left|s_{n}-s_{n-1}\right| \leq 1, \quad\left|s_{n-1}-s_{n-2}\right|>2 \cdots \cdots
\end{aligned}
$$
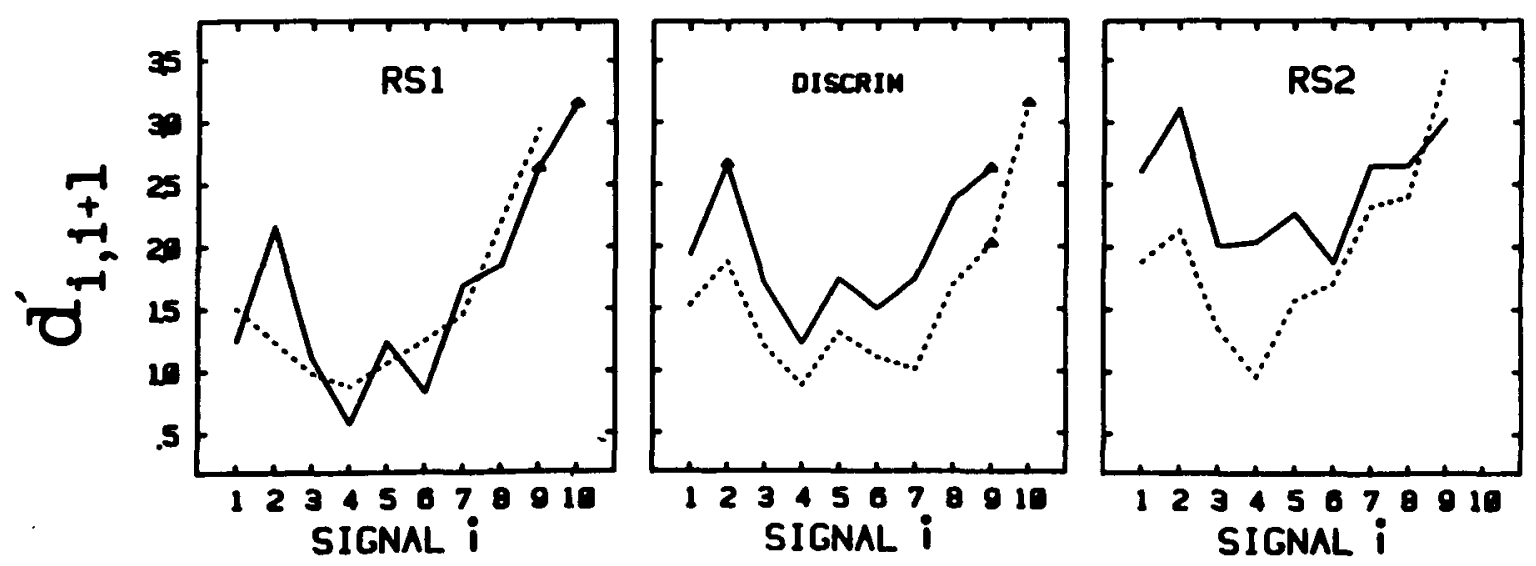

Figure 2. Values of $d^{\prime}{ }_{i, i+1}$ as a function of signal $i$ for RS1, the discrimination condition and RS2, after conditioning according to the previous two signals. Points marked with a filled triangle are based on only one of a possible two $d^{\prime}{ }_{i, j+1}$ estimates. Note also that there are several instances of missing $d^{\prime}{ }_{10,11}$ values due to ceiling effects. 
on roughly $1 / 3$ of the trials. In a third condition, each of the extreme intensities was presented on roughly $1 / 5$ of the trials. Although the subjects exhibited shifts in response bias in the expected directions, Chase et al. found that manipulating the a priori probability of signal presentations did not affect sensitivity significantly.

This result is not what one would expect if there is an attention band that tracks signal presentations probabilistically. Furthermore, in view of the sequential effects reported in Experiment 1 of this article, it seems reasonable to expect that increasing the a priori probability of a given signal should improve sensitivity in the region of that signal. For example, assume that one increases the probability of presenting signal $i$; then there will be an increased proportion of instances in which signal $i$ is presented on successive trials or in which signals $i$ and $i+1$ are presented on successive trials.

On the basis of the above-mentioned findings and considerations, it seemed reasonable to run an $\mathrm{AI}$ experiment in which the a priori probability of signal presentations was manipulated. This was one of the purposes of Experiment 2. A second purpose was to gain preliminary evidence that subjects could voluntarily control the location of the attention band.

\section{Method}

The signal set, apparatus, and subjects were the same as in Experiment 1. Unless otherwise mentioned, all other aspects of the procedures were also identical.
The subjects participated in two conditions on roughly alternating days. In condition $U$, the a priori presentation probabilities for each signal were uniformly distributed-that is, this was a standard, random-step AI experiment. In condition $\mathbf{M}$, the a priori probability of signal $S$ was $1 / 4$, and the a priori probability of signal 6 was $1 / 4$. All the remaining stimuli were equally likely. The subjects were informed as to the nature of these manipulations.

Each subject completed 3,800 trials in condition $U$ and 3,900 trials in condition $\mathbf{M}$, all of which are included in the analyses. About 9 or 10 blocks of data were collected per session for each subject.

\section{Results and Discussion}

A Thurstone Case V (constant-variance) model was applied to the cumulative judgment matrices for each subject in both conditions. A standard parameter search routine with a maximum-likelihood criterion was utilized to obtain the stimulus distribution and category boundary means. Two starting configurations were used for each parameter search. The first was the algebraic solution described by Torgerson (1958), and the second was obtained by using the pairwise $d^{\prime}{ }_{i, i+1}$ procedure employed throughout Experiment 1 . Regardless of starting configuration, the search routine converged to the identical final solution in all cases. It should also be noted that the pairwise $d^{\prime} i, i+1$ procedure and the Thurstone Case $V$ analysis yielded similar patterns of results for these data. The Case V analysis is reported here because it is more traditional.

The results on sensitivity are plotted in Figure 3 for each subject separately. The solid lines corre-

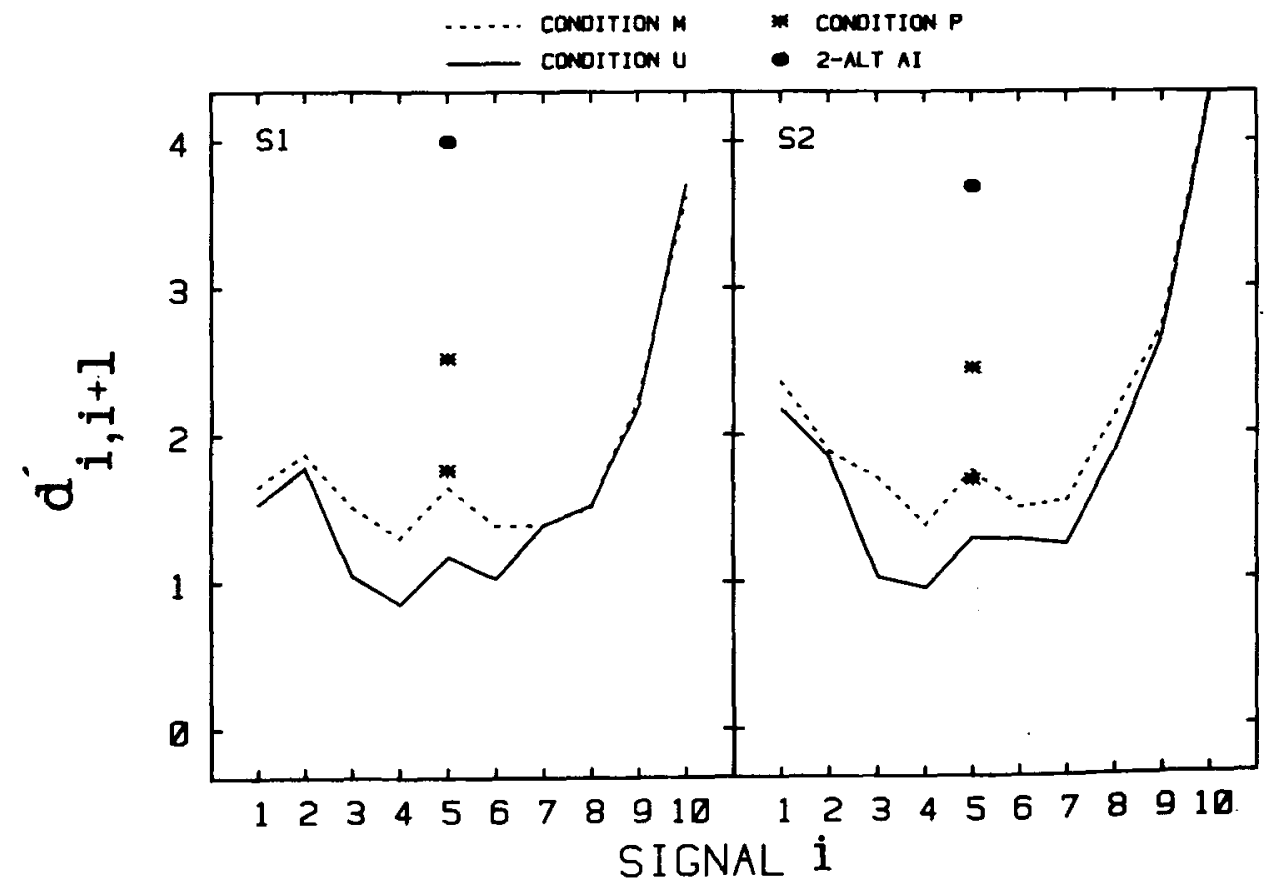

Figure 3. Values of $d^{\prime}{ }_{1,1+1}$ as a function of signal $I$ for conditions $U, M$, and $P$ and the two-alternative A condition. 
spond to the $d^{\prime}{ }_{i, i+1}$ values obtained in condition $U$, and the dashed lines correspond to the $d^{\prime}{ }_{i, i+1}$ values obtained in condition $M$. (The reader should ignore, for present purposes, the other symbols that appear on the figure.).

Sensitivity is improved for both subjects in the vicinity of the center signals in condition $M$ relative to condition $U$. As one moves further away from the center, sensitivity in the two conditions converges. This observation is confirmed by statistical test. Using the significance test proposed by Gourevitch and Galanter (1967) for differences in $d^{\prime}$, one obtains the following results. For $\mathrm{S1}$, performance on $d^{\prime}$ pairs 3-4, 4-5, 5-6, and 6-7 is significantly better in condition $M$ than in condition $U(p<.05)$. For $S 2$, performance on $d^{\prime}$ pairs $3-4,4-5$, and $5-6$ is significantly better in condition $\mathbf{M}$ than in condition $\mathbf{U}$ $(p<.05)$. No other differences are significant at the .05 level for either subject.

These results are only somewhat consistent with the attention-band theory of Luce et al. (1976). Since there are more presentations of the center signals in condition $\mathrm{M}$ relative to condition $\mathrm{U}$, there should also be a higher probability of the attention band's shifting to this region of the intensity continuum and thereby improving sensitivity. Nevertheless, one would also expect decreased sensitivity towards the edges of the stimulus set in condition $M$ relative to condition $U$, rather than identical sensitivity.

Voluntary manipulation of attention? Thus far, improved sensitivity in the various experiments seems to have been mainly under the control of sequential dependencies in signal presentations. According to the attention-band theory, this is the result of attention's tending to track signal presentations. Previous experiments have used differential payoffs in attempts to manipulate the voluntary focusing of attention (Lippmann, Braida, \& Durlach, 1976; Weber, Green, \& Luce, 1977). These attempts have failed-sensitivity in unidimensional absolute identification tasks appears to be independent of payoffs.

A more extreme approach is applied in Experiment 2 of the current study. Upon completion of conditions $U$ and $M$, both subjects were run for 2 days in an additional condition, denoted $P$. The same stimulus presentation schedule was maintained in condition $\mathbf{P}$ as appeared in condition $\mathbf{M}$. The task requirements in condition $P$ were changed, however. In particular, the subjects were required to perform a "partial identification" task. Whenever a stimulus greater than or equal to 6 was presented, the subjects were asked to respond 6; whenever a stimulus less than or equal to 5 was presented, the subjects were asked to respond 5. Feedback on each trial consisted of whether the stimulus was greater than or equal to 6 , or less than or equal to 5 .

Subjects would perform maximally in this experiment by focusing attention on the center signals, 5 and 6 . This experiment may be viewed as a case in which the most extreme form of payoffs were employed. It was only of value to the subjects to distinguish between the set of signals less than or equal to 5 , and the set of signals greater than or equal to 6. All other distinctions were of zero value.

There are independent grounds for expecting that subjects' ability to resolve signals 5 and 6 would improve in condition $\mathbf{P}$ relative to condition $\mathbf{M}$. Gravetter and Lockhead (1973) have proposed that the variability in signal representations is directly related to criterial range, not stimulus range. Criterial range is defined as the distance between the two extreme category boundaries utilized by the subject in his or her recognition judgments. In condition $P$, the criterial range is effectively zero, since only a single category boundary need be maintained. Gravetter and Lockhead's theory would seem to make the extreme prediction that sensitivity in distinguishing signals 5 and 6 in condition $P$ would be the same as in a two-alternative $\mathrm{AI}$ task, since criterial range would equal zero in both cases. Following completion of condition $P$, therefore, the subjects were tested in a two-alternative AI task using only signals 5 and 6 , each signal presentation being random.

On Day 1 of testing in condition $P, 1,400$ responses were collected for each subject. On Day 2 of testing in condition $P, 1,100$ responses were collected for each subject. Five hundred responses were then obtained for each subject in the two-alternative AI task. All other aspects of the procedures were the same as already described for conditions $U$ and $M$.

The analysis is based on those trials in condition $P$ for which either of signals 5 and 6 were presented. The values of $d^{\prime}{ }_{5,6}$ obtained on each day of testing are presented for each subject in Figure 3 (asterisks). The lower asterisk in each graph corresponds to the $d^{\prime}{ }_{5,6}$ value obtained on Day 1 , and the higher asterisk to the $d^{\prime} s, 6$ value obtained on Day 2. The filled circles are the $d^{\prime}{ }_{5,6}$ values obtained for each subject in the two-alternative AI task.

Inspection of the figure reveals virtually identical performance on Day 1 in condition $P$ and in condition $M$. Nevertheless, by Day 2 there is marked improvement in both subjects' ability to distinguish signals 5 and 6 in condition $P$. The subjects still have a long way to go, however, compared with the twoalternative situation.

In conclusion, it appears that the changed task requirements in condition $P$ resulted in improved sensitivity in distinguishing signals 5 and 6 , although the behavior took some time to develop. One interpretation of these findings is that the subjects learned to focus the attention band on the center signals to a greater extent, although other interpretations are available. For example, the subjects may have been able to develop an improved memory representation for the category boundary partitioning the center signals. 


\section{EXPERIMENT 3}

The experimental manipulations described thus far in this article were all embedded in one-interval paradigms. These are paradigms in which the stimulus on each trial is an individually presented signal. Also of interest are the two-interval paradigms; in these, the stimulus on each trial is a temporally ordered pair of signals. The two-interval paradigms are typically discrimination experiments in which the subject must make a binary judgment such as louder or softer. Durlach and Braida (1969) have argued that the paradigm of particular value in studying purely discrimination processes (as opposed to ability to identify overall level) is the two-interval rovinglevel discrimination paradigm. In this paradigm, the subject is required to judge whether the second signal presented on each trial is louder or softer than the first signal, but the overall level of the signals is varied between trials.

The purpose of Experiment 3 was to demonstrate the existence of sequential effects in a roving-level discrimination paradigm-effects that may be explained in natural fashion by the hypothesis of a shifting attention band. Schematically, the design of Experiment 3 was as follows. The stimulus set consists of two clusters of intensities-one cluster of relatively low intensities, and one cluster of relatively high intensities. The subjects were first required to make a series of 10 discrimination judgments with signals from one of the intensity clusters, for example, intensity cluster 1 . Then, they were required to make another series of 10 discrimination judgments with signals from intensity cluster 2 . Then, once again, they made 10 discrimination judgments with signals from cluster 1 , and so forth in this alternating fashion. The intuition underlying this experiment was that subjects would perform maximally by shifting the attention band (either automatically or voluntarily) to the intensity cluster from which signals were being presented. When the cluster was switched, however, attention would be focused in the wrong location. The hypothesis, then, was that one should observe improvement in discrimination performance as the trial sequence number of a given cluster increases (where the trial sequence number varies from 1 to 10 ).

\section{Method}

The apparatus was the same as that already described for Experiments 1 and 2. Three new subjects were hired. The author participated as a fourth subject.

The experiment was organized into blocks of 100 trials, with rests between blocks. Typically, nine blocks of trials were completed per 2-h session.

The stimulus set consisted of two clusters of seven intensities each. In the soft cluster, the seven intensities were spaced in $\mathbf{7 5}$ $\mathrm{dB}$ increments from 42.75 to $47.25 \mathrm{~dB}$ SPL. In the loud cluster, the seven intensities were spaced in .75-dB increments from 72.75 to $77.25 \mathrm{~dB}$ SPL. (For the author, the loud cluster inten- sities ranged from 82.75 to $87.25 \mathrm{db}$ SPL.) On the first trial of each block, a random decision was made as to which intensity cluster would be presented first. Ten consecutive discrimination trials were then presented with signals from this cluster. Then 10 consecutive discrimination trials were presented with signals from the other cluster, and so forth.

On a given trial, a random integer $i$ between 2 and 6 (inclusive) was selected. Stimulus i from the current cluster was the first signal presented on the trial. A random selection was made as to whether stimulus $i-1$ or $i+1$ was then presented as the second signal on the trial. The subjects were required to judge whether the second signal was louder than the first (response 2) or softer than the first (response 1).

The signal duration was $500 \mathrm{msec}$. There was a 500 -msec ISI between the two signals presented on a trial. As soon as all subjects had entered a response, feedback was provided for 500 msec. Each trial was preceded by a 500 -msec warning interval during which the word "READY" was printed on the screen.

An average of 7,375 total responses was collected per observer, or 369 responses per trial sequence number per cluster per observer. The hired subjects were paid $\$ 3.50$ per hour for participating in the experiment.

\section{Results and Discussion}

The analysis looks at each subject's overall percent correct for each trial sequence number for each separate cluster, cumulating over the individual signals within a cluster. I use percent correct as the performance measure for this experiment rather than d' because, due to certain response biases (see next subsection), some subjects never committed "misses" or "false alarms" on some signal pairs. The results are plotted in Figure 4. As hypothesized, there is improvement in performance as the trial sequence number increases, with most of the improvement coming relatively early in the sequence. There are also hints of a drop in performance very late in the trial sequence ( $S 1$ and $S 2$ in the loud cluster). This was not totally unexpected-it may correspond to subjects' beginning to switch attention in preparation for hearing intensities from the opposite cluster.

Response-bias effects. Nevertheless, it should also be pointed out that there are large and consistent response-bias effects in the data that the attentionband model itself cannot explain. These are plotted in Figure 5. On the abscissa is the value of the initial signal presented on a given trial. The solid curves correspond to those trials in which the second signal was louder than the first, and the dashed curves to those trials in which the second signal was softer than the first. The filled triangles are the loud cluster data, and the open circles are the soft cluster data.

The response-bias effect is that, within each cluster, subjects have a tendency to respond "louder" when the signals presented are at the upper edge of the cluster, whereas they have a tendency to respond "softer" when the signals are from the lower edge of the cluster. This phenomenon has been reported previously by Berliner, Durlach, and Braida (1977) for roving-level discrimination experiments in which the signal levels are uniformly distributed 


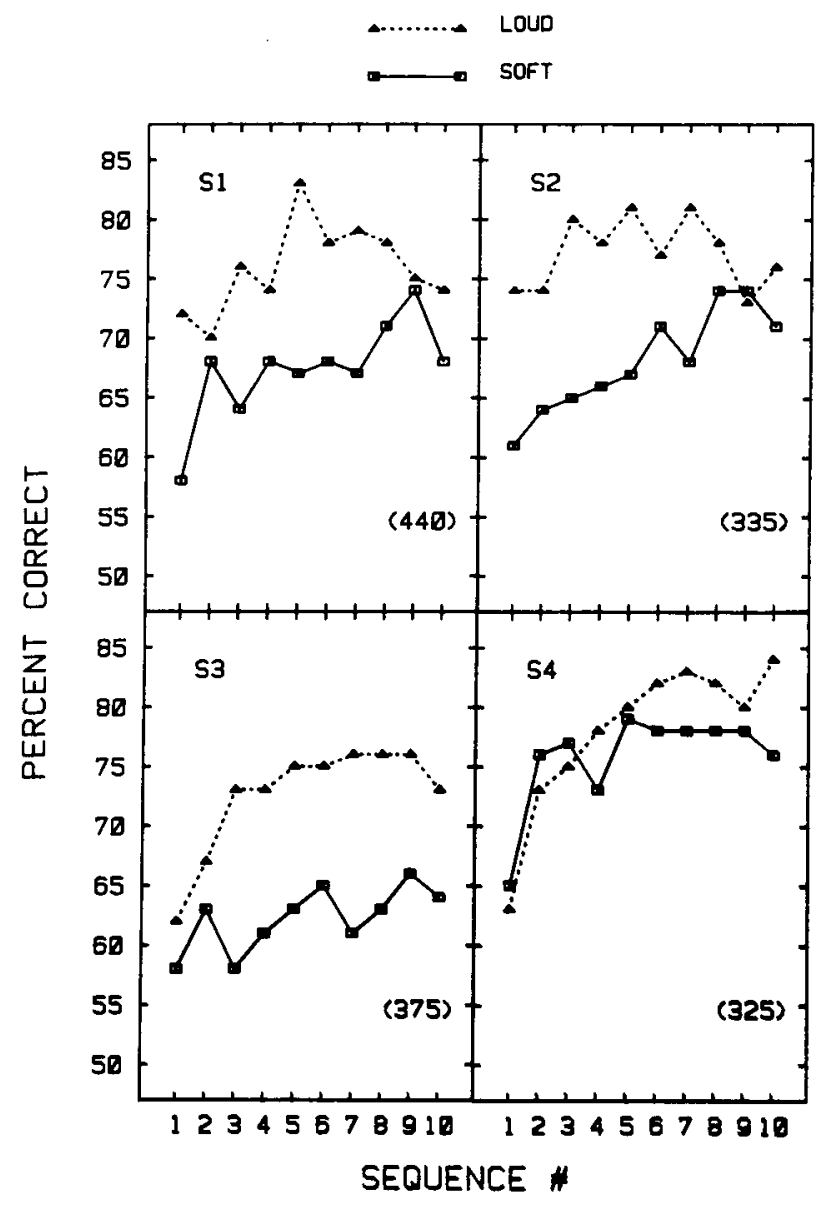

Figure 4. Percent correct as a function of trial sequence number in the sequentially constrained roving-level discrimination experiment. Values in parentheses are the number of observations upon which each data point is based.

across the range. They have termed it the "bias edge effect." The finding in the present study is that under the extreme sequential dependencies employed, each individual cluster yielded its own bias edge effect.

\section{CONCLUSIONS}

The results of the experiments reported in this article supply converging evidence that subjects' ability to resolve stimuli lying along a unidimensional continuum is influenced by sequential dependencies in stimulus presentations. Changes in sensitivity were observed in an AI experiment in which sequential dependencies were manipulated, in an $\mathrm{AI}$ experiment in which a priori presentation probability was manipulated, and in a roving-level discrimination experiment in which sequential dependencies were manipulated. The results are consistent with the theory of Luce, Green, and Weber (1976), which posits an attention band that tracks signal presentations probabilistically. The results challenge theories that as- sume that the variance of signal representations in absolute identification is a function solely of stimulus range (Durlach \& Braida, 1969).

The possibility that the subjects' vastly improved performance on the small-step AI design was due solely to their use of a discrimination strategy, which is not normally used in a random-presentation task, was ruled out. In particular, changing the task requirements of the random-step identification design such that the subjects simply had to make directional judgments from one trial to the next left resolution performance virtually unchanged. In addition, two-trial sequential analyses performed on the random-step data yielded converging evidence that the cumulative nature of the sequential dependencies in the small-step condition was of fundamental importance in improving the subjects' sensitivity.

Preliminary evidence that subjects can voluntarily focus attention at a given intensity region was obtained. The subjects' ability to distinguish signals 5 and 6 in Experiment 2 improved markedly when they were no longer required to make all the additional discriminations along the intensity continuum. A similar result has been reported by Weber

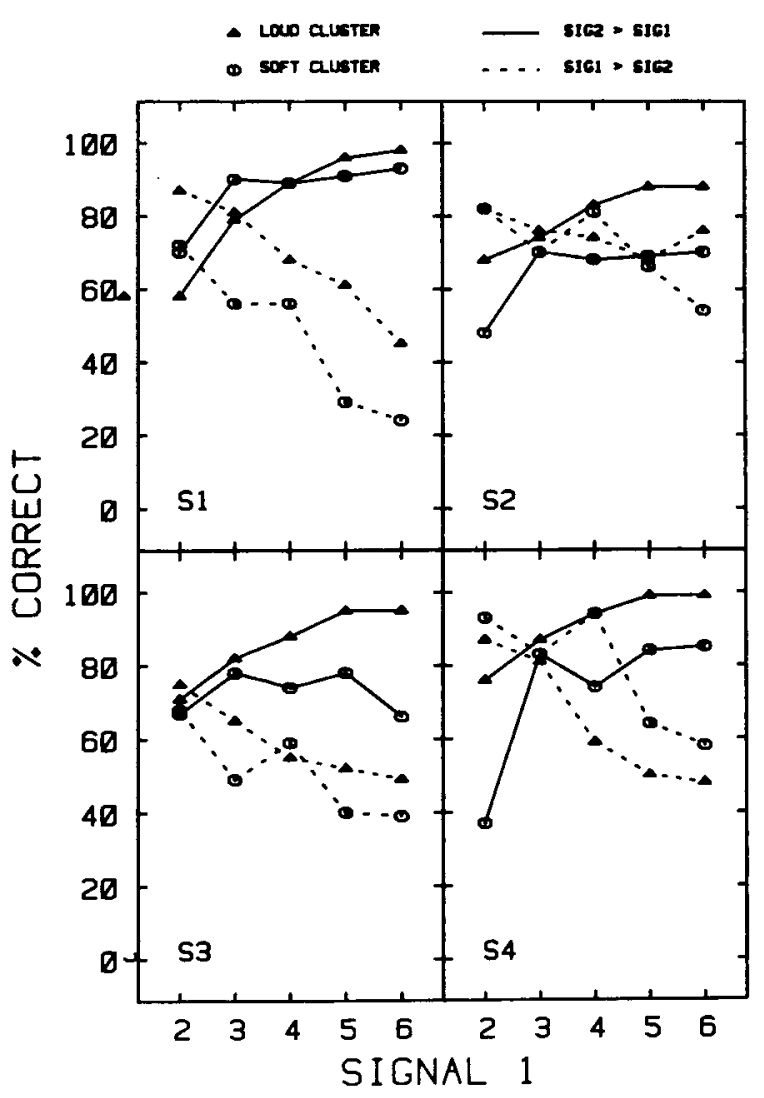

Figure 5. Response bias effect in the roving-level discrimination experiment. The blas effect is for the subject to respond "louder" for signals at the upper edge of each intensity cluster, and to respond "softer" at the lower edge of each intensity cluster. 
et al. (1977). These findings are interesting because other attempts to manipulate the voluntary focusing of attention using differential payoffs have failed (Lippmann et al., 1976; Weber et al., 1977). The contrasting findings may suggest that subjects cannot voluntarily divide attention along several locations of a unidimensional continuum, but that they can attend to one location in an all-or-none manner.

Finally, why are two signals that are almost perfectly identifiable in a two-alternative AI task confused when embedded in a multisignal task? Part of the answer may lie in the limited attentional capacities of human observers. As demonstrated in Luce et al. (1982) and in the present article, the inferior performance is ameliorated when sequential dependencies are arranged to allow attention to gradually track signal presentations, and in situations in which subjects are enabled to voluntarily attend to the relevant signal pair.

\section{REFERENCES}

Berlinen, J. E., Durlach, N. I., \& Braida, L. D. Intensity perception. VII. Further data on roving-level discrimination and the resolution and bias edge effects. Journal of the Acoustical Society of America, 1977, 61, 1577-1585.

Braida, L. D., \& Durlach, N. I. Intensity perception. II. Resolution in one-interval paradigms. Journal of the Acoustical Society of America, 1972, 51, 483-502.

Chase, S., Bugnacki, P., Braida, L. D., \& Durlach, N. I. Intensity perception. XII. Effect of presentation probability on absolute identification. Journal of the Acoustical Society of America, 1983, 73, 279-284.

Durlach, N. I., \& Braida, L. D. Intensity perception. 1. Preliminary theory of intensity resolution. Journal of the Acoustical Society of America, 1969, 46, 372-383.

Gourevitch, V., \& Galanter, E. A significance test for one parameter isosensitivity functions. Psychometrika, 1967, 32, 25-33.

Gravetter, F., i Lockhead, G. R. Criterial range as a frame of reference for stimulus judgment. Psychological Review, 1973, 80, 203-216.

Hartman, E. B. The influence of practice and pitch-distance between tones on the absolute identification of pitch. American Journal of Psychology, 1954, 67, 1-14.

Lippmann, R. P., Braida, L. D., \& Durlach, N. I. Intensity perception. V. Effect of payoff matrix on absolute identification. Journal of the Acoustical Society of America, 1976, 59, 129-134.

Luce, R. D., Green, D. M., \& Weber, D. L. Attention bands in absolute identification. Perception \& Psychophysics, 1976, 20, 49-54.

Luce, R. D., Nosofsky, R. M., Green, D. M., \& Smith, A. F. The bow and sequential effects in absolute identification. Perception \& Psychophysics, 1982, 32, 397-408.

Pollack, I. The information of elementary auditory displays. I. Journal of the Acoustical Society of America, 1952, 24, 745-749.

Purks, S. R., Callahan, D. J., Braida, L. D., \& Durlach, N. I. Intensity perception. X. Effect of preceding stimulus on identification performance. Journal of the Acoustical Society of America, 1980, 67, 634-637.

Siegel, W. Memory effects in the method of absolute judgment. Journal of Experimental Psychology, 1972, 94, 121-131.

Toraerson, W. R. Theory and methods of scaling. New York: Wiley, 1958.

Weber, D. L., GreEN, D. M., Luce, R. D. Effects of practice and distribution of auditory signals on absolute identification. Perception \& Psychophysics, 1977, 22, 223-231.

\section{NOTES}

1. The improved sensitivity in the small-step condition is not a measurement artifact of the restricted response choices on each trial. The method of analysis used to compare performance in the small-step and random-step conditions controlled for this factor (see Luce et al., 1982).

2. As noted by an anonymous referee, this is not expected to be the case for any given finite session. Nevertheless, it should be noted that the subjects were presented with a substantial number of signal presentations from the entire range in all sessions tested.

3. The reader should note that a given response may be defined differently depending on the $d$ ' pair that is being calculated. For example, assume in the identification condition that stimulus 6 is presented and the subject responds 7 . This is considered a "hit" in the calculation of d' 5,6 , but a "false alarm" in the calculation of $d^{\prime}{ }_{6,7}$. Similarly, assume in the discrimination condition that stimulus 6 is presented on trial $n$ and that it is repeated on trial $n+1$. A response of "louder" is considered a "hit" in the calculation of $d{ }^{3,6}$, but a "false alarm" in the calculation of $\mathrm{d}^{\prime}{ }_{6,7}$.

4. The proper method of analysis in this situation is to obtain separate estimates of $d^{\prime}$ contingent on $S_{n-1}=i$ and $S_{n-1}=i+1$, and then average these together (as was done previously). This is because there are strong first-order effects on response bias in these tasks-subjects have a tendency to repeat the response that was correct on the previous trial (Luce et al., 1982; Purks et al., 1980). Cumulating over the opposite patterns of response bias and then estimating $d^{\prime}$ may cloud the main effects of interest. Unfortunately, the preferred method of analysis can be used only in RSI and in the discrimination condition; small sample sizes force me to use the flawed cumulative analysis for RS2.

(Manuscript received June 21, 1982; revision accepted for publication November $29,1982$. ) 